Check for updates

Cite this: Phys. Chem. Chem. Phys., 2020, 22, 17597

Received 26th February 2020 Accepted 30th June 2020

DOI: $10.1039 / \mathrm{d} 0 \mathrm{cp01110j}$

rsc.li/pccp

\section{Frame-invariant Fick diffusion matrices of multicomponent fluid mixtures}

\author{
José M. Ortiz de Zárate ${ }^{a}$ and Jan V. Sengers* ${ }^{b}$ \\ Extension of a description of mass diffusion in binary fluids based on Fick's law to multicomponent fluids \\ requires introduction of diffusion matrices. A problem is that Fick diffusion matrices commonly adopted \\ for multicomponent fluids depend on the velocity frame of reference. In this paper we show how one \\ can define Fick diffusion matrices for multicomponent fluids that are frame invariant.
}

\section{Introduction}

Diffusion in multicomponent fluid mixtures is a ubiquitous phenomenon in nature and in technical applications. While diffusion in binary mixtures is well understood, a better understanding of diffusion in multicomponent fluids is still desirable, in particular regarding cross-diffusion coefficients and their dependence on reference frames. For this reason there are currently extensive efforts, involving several research groups, for an accurate characterization of diffusion coefficients (including cross-diffusion) for selected liquid mixtures, starting with ternary mixtures as a stepping stone towards fully multicomponent fluid mixtures. ${ }^{1-5}$

Because of the phenomenon of cross-diffusion, ${ }^{6}$ the description of mass diffusion in multicomponent mixtures is qualitatively different from the simpler case of a binary mixture. Even in the case of isotropic mixtures, to be considered in the present paper, the mathematical description of diffusion requires diffusion matrices. A major complication is that the actual values of coefficients in diffusion matrices based on Fick's law depend upon the representation used to specify the composition of the mixture, i.e., whether mass fractions, mole fractions or volume fractions are used. As will be discussed in Section 2, a specific choice of concentration representation correlates with a specific frame of reference for the definition of diffusion fluxes. This frame dependence of the diffusion coefficients does not exist in isotropic binary mixtures, for which a single coefficient has the same numerical value, regardless of the concentration representation adopted. But the frame dependence

\footnotetext{
${ }^{a}$ Department of Structure of Matter, Thermal Physics and Electronics, Complutense University, 28040 Madrid, Spain. E-mail: jmortizz@ucm.es

${ }^{b}$ Institute for Physical Science and Technology, University of Maryland,

College Park, MD 20742, USA. E-mail: sengers@umd.edu
}

of Fick diffusion matrices introduces complexity and confusion in diffusion research in multicomponent mixtures. Sometimes authors do not specify the frame of reference adopted in reporting experimental data and leave it up to the reader to infer the frame of reference from the experimental technique used to get the diffusion-coefficient data. ${ }^{7-9}$ A detailed analysis of the complexities associated with the frame dependence of the diffusion matrices in ternary mixtures has recently been reported by Svetsova and coworkers. ${ }^{10}$

Some of the problems associated with Fick diffusion matrices may be alleviated by adopting the Maxwell-Stefan approach to diffusion in multicomponent mixtures. ${ }^{6,11-15}$ Maxwell-Stefan diffusion matrices relate chemical-potential gradients to differences between velocities of the components and the resulting diffusion matrices do not depend on a reference frame. ${ }^{13-15}$ Nevertheless, Fick's approach to diffusion is most popular in experiments, because chemical potentials in a multicomponent mixture can only be obtained indirectly, while concentrations allow for direct measurement. Fick's approach also combines more naturally with mass balance in multicomponent fluid flow. ${ }^{16,17}$

The problem of frame dependence, similar to the one appearing in Fick's approach for isothermal diffusion, is also encountered in the case of thermodiffusion. ${ }^{18}$ In the case of thermodiffusion, the issue already requires a specific frameindependent definition of the thermodiffusion coefficient $D_{\mathrm{T}}$ or Soret coefficient $S_{\mathrm{T}}$ for binary mixtures. In a previous paper one of us has shown how the procedure for binary mixtures can be generalized to obtain frame-independent thermodiffusion coefficients of multicomponent mixtures. ${ }^{19}$ In the present paper we consider the case of isothermal diffusion that was not considered in the previous paper. Specifically, we shall show how a simple similarity transformation of frame-dependent Fick diffusion matrices yields Fick diffusion matrices that are indeed independent of the reference frame. 
We have organized this paper as follows. First and for completeness, we start in Section 2 by reviewing different frames of references and corresponding diffusion fluxes. Then, in Section 3, we review Fick's diffusion in binary mixtures, emphasizing the frame independence of the single diffusion coefficient $D$. We then proceed to consider in Section 4 the case of a ternary mixture, which represents the first type of mixture where the complications associated with frame dependence of Fick's diffusion matrices first appears, followed by an analysis of binary limits in Section 5. In Section 6 we make some comments on the composition dependence of frame-dependent versus frame-independent diffusion matrices for a particular example of a ternary mixture. In Section 7 we show how the procedure, specified in detail for ternary mixtures, can be readily generalized to multicomponent mixtures. In Section 8 we show how the results of the present paper for isothermal diffusion can be extended to the general case of non-isothermal diffusion. Our conclusions are summarized in Section 9.

\section{Diffusion fluxes}

This paper is primarily concerned with isothermal diffusion caused by the presence of a concentration gradient. Comments on thermodiffusion resulting from the presence of a temperature gradient will be added at the end of the paper. Diffusion appears because, in general, in a multicomponent mixture different components $i$ move with different velocities $\boldsymbol{u}_{i}$. The diffusion flux $\boldsymbol{J}_{i}$ of component $i$ is generally defined as proportional to the difference between the velocity $\boldsymbol{u}_{i}$ and some average velocity. Depending on the choice adopted for the average velocity, different diffusion fluxes can be defined, namely, relative to the average molar velocity, the barycentric velocity, or the average volume velocity. The following three diffusion fluxes are most used in practice: ${ }^{6}$

(1) Mass diffusion flux relative to the center of mass velocity:

$$
\boldsymbol{J}_{i}^{\mathrm{w}}=\rho_{i}\left[\boldsymbol{u}_{i}-\left(\sum_{j} w_{j} \boldsymbol{u}_{j}\right)\right]
$$

in terms of SI units of $\mathrm{kg} \mathrm{m}^{-2} \mathrm{~s}^{-1}$. Here $\rho_{i}$ is the mass density of component $i\left(\mathrm{~kg} \mathrm{~m}^{-3}\right)$ and $w_{i}=\rho_{i} / \rho_{\mathrm{t}}$ the corresponding mass fraction with $\rho_{\mathrm{t}}=\sum_{j} \rho_{j}$ the total mass density. In this paper we adopt the convention that the summation is over all components of the mixture unless indicated otherwise.

(2) Molar diffusion flux relative to the center of molar velocity:

$$
\boldsymbol{J}_{i}^{\mathrm{x}}=c_{i}\left[\boldsymbol{u}_{i}-\left(\sum_{j} x_{j} \boldsymbol{u}_{j}\right)\right]
$$

in terms of SI units of mol m $\mathrm{m}^{-2} \mathrm{~s}^{-1}$. Here $c_{i}$ is the molar concentration of component $i\left(\mathrm{~mol} \mathrm{~m}^{-3}\right)$ and $x_{i}=c_{i} / c_{\mathrm{t}}$ the corresponding mole fraction with $c_{\mathrm{t}}=\sum_{j} c_{j}$ the total molar density (equal to the inverse molar volume).
(3) Molar diffusion relative to the center of volume velocity:

$$
\boldsymbol{J}_{i}^{\mathrm{V}}=c_{i}\left[\boldsymbol{u}_{i}-\left(\sum_{j} \phi_{j} \boldsymbol{u}_{j}\right)\right]
$$

in terms of SI units of mol m${ }^{-2} \mathrm{~s}^{-1}$. Here $\phi_{i}=x_{i} \hat{V}_{i} / \sum_{j} x_{j} \hat{V}_{j}$ is the volume fraction of component $i$, where $\hat{V}_{i}$ is the partial molar volume of component $i$. The total molar volume is $\hat{V}=$ $\sum_{j} x_{j} \hat{V}_{j}$ (equal to the inverse total molar concentration).

For the purpose of this paper it is important to distinguish among the different diffusion fluxes and properties defined in the different reference frames, for which we use roman superscripts, w, x, or V, as we did in the equations above. According to eqn (1)-(3), one can define for each reference frame as many diffusion fluxes as the number of components $n$ in the mixture. However, only $n-1$ of these fluxes are independent, since: ${ }^{6}$

$$
\sum_{i} \boldsymbol{J}_{i}^{\mathrm{w}}=0, \quad \sum_{i} \boldsymbol{J}_{i}^{\mathrm{x}}=0, \quad \sum_{i} \boldsymbol{J}_{i}^{\mathrm{V}}=0
$$

Note that $c_{\mathrm{t}}^{-1}=\sum_{j} x_{j} \hat{V}_{j}$.

\section{Isothermal Fick diffusion in binary mixtures}

In an binary mixture there is only one independent concentration, such as a mass fraction $w$ or a mole fraction $x$, and, in accordance with eqn (4), one single independent diffusion flux. Upon solving eqn (1) and (2) for $\boldsymbol{u}_{1}-\boldsymbol{u}_{2}$, the relationship between the single independent mass diffusion flux $\left(\right.$ e.g., $\left.\boldsymbol{J}^{\mathrm{W}}=\boldsymbol{J}_{1}^{\mathrm{W}}\right)$ and the single independent molar diffusion flux $\left(e . g ., \boldsymbol{J}^{\mathrm{x}}=\boldsymbol{J}_{1}^{\mathrm{x}}\right)$ is readily expressed as: $:^{6,19}$

$$
\frac{\boldsymbol{J}^{\mathrm{w}}}{\rho_{\mathrm{t}} w(1-w)}=\frac{\boldsymbol{J}^{\mathrm{x}}}{c_{\mathrm{t}} x(1-x)} .
$$

In nonequilibrium thermodynamics ${ }^{13,20,21}$ Fick's law postulates a linear relationship between the mass flux and the gradient of mass fraction or the molar diffusion flux and the gradient of mole fraction. For isotropic binary mixtures:

$$
\begin{gathered}
\boldsymbol{J}^{\mathrm{w}}=-\rho_{\mathrm{t}} D \boldsymbol{\nabla} \boldsymbol{w}, \\
\boldsymbol{J}^{\mathrm{x}}=-c_{\mathrm{t}} D \boldsymbol{\nabla} x .
\end{gathered}
$$

For the purpose of the present paper it is important to note that, in a binary mixture, the relationship between the gradient of mass fraction and the gradient of mole fraction can be expressed as ${ }^{6,19,22}$

$$
\frac{\nabla w}{w(1-w)}=\frac{\nabla x}{x(1-x)} .
$$

From eqn (5) and (7) it follows that the two relations in eqn (6) are perfectly consistent, i.e., the single scalar diffusion coefficient $D$ of an isotropic binary mixture is invariant under change from 
the barycentric frame of reference of eqn (1) to the center of molar-velocity frame of reference of eqn (2).

This is also true for the volume frame of reference. On the one hand, upon solving eqn (2) and (3) for $\boldsymbol{u}_{1}-\boldsymbol{u}_{2}$, the relationship between the different diffusion fluxes can be expressed as ${ }^{6}$

$$
\frac{\boldsymbol{J}_{1}^{\mathrm{V}}}{x(1-\phi)}=\frac{\boldsymbol{J}^{\mathrm{x}}}{x(1-x)}=\frac{-\boldsymbol{J}_{2}^{\mathrm{V}}}{\phi(1-x)},
$$

where use has been made of the relation between molar concentrations and molar fractions, namely, $c_{1}=x c_{\mathrm{t}}$ and $c_{2}=(1-x) c_{\mathrm{t}}$. On the other hand, the relationship between gradients in molar concentration and mole fraction of component 1 for a binary mixture can be expressed as ${ }^{6}$

$$
\begin{aligned}
& \boldsymbol{\nabla} c_{1}=\frac{\hat{V}_{2}}{\hat{V}} \boldsymbol{\nabla} x=\frac{1-\phi}{1-x} c_{\mathrm{t}} \boldsymbol{\nabla} x, \\
& \boldsymbol{\nabla}_{c_{2}}=-\frac{\hat{V}_{1}}{\hat{V}} \boldsymbol{\nabla} x=-\frac{\phi}{x} c_{\mathrm{t}} \boldsymbol{\nabla} x .
\end{aligned}
$$

Upon substituting both eqn (8) and (9) into eqn (6), one readily obtains Fick's law in the volume frame of reference:

$$
\begin{aligned}
& \boldsymbol{J}_{1}^{\mathrm{V}}=-D \boldsymbol{\nabla} c_{1}, \\
& \boldsymbol{J}_{2}^{\mathrm{V}}=-D \boldsymbol{\nabla} c_{2},
\end{aligned}
$$

with the same diffusion coefficient $D$ that appears in eqn (6).

The consistency of eqn (6) and (10) confirms the frame invariance of isothermal Fick's diffusion in a binary mixture. This is an important property from a practical point of view. It makes dealing with isothermal Fick diffusion in binary mixtures simpler, whether theoretically, ${ }^{20}$ experimentally, ${ }^{1,3,4,7,8,23}$ or by numerical simulations. ${ }^{24,25}$ Thus scientists can continue to use their preferred concentration representation, either mass fraction, ${ }^{1,4,5,20}$ mole fraction,,$^{7,8,24,25}$ or molar concentration ${ }^{23,26}$ without a need for inconvenient conversion to compare values reported for the diffusion coefficient of binary mixtures.

Eqn (6) and (10) also show how each reference frame for the definition of diffusion fluxes naturally correlates with a specific concentration representation, either mass fraction, mole fraction, or molar concentration.

\section{Isothermal Fick diffusion in ternary mixtures}

In ternary mixtures, in accordance with eqn (4), there are two independent diffusion fluxes and, associated with them, two independent concentration gradients. A description of isothermal diffusion by Fick's law assumes all components to be equal, so that a given diffusion flux depends linearly on all independent concentration gradients. In the barycentric frame of reference, the first relation in eqn (6) is usually extended to a ternary mixture by writing ${ }^{6}$

$$
\left(\begin{array}{c}
\boldsymbol{J}_{1}^{\mathrm{w}} \\
\boldsymbol{J}_{2}^{\mathrm{w}}
\end{array}\right)=-\rho_{\mathrm{t}} \mathrm{D}^{\mathrm{w}} \cdot\left(\begin{array}{c}
\boldsymbol{\nabla} w_{1} \\
\boldsymbol{\nabla} w_{2}
\end{array}\right)
$$

with

$$
\mathrm{D}^{\mathrm{W}}=\left[\begin{array}{ll}
D_{11}^{\mathrm{w}} & D_{12}^{\mathrm{w}} \\
D_{21}^{\mathrm{w}} & D_{22}^{\mathrm{w}}
\end{array}\right],
$$

the Fick diffusion matrix in the barycentric frame of reference. In the center of molar-velocity frame of reference, one expresses Fick's law with concentrations in terms of mole fraction and, similarly to the second relation in eqn (6) for binary mixtures, one writes for a ternary mixture

$$
\left(\begin{array}{c}
\boldsymbol{J}_{1}^{\mathrm{x}} \\
\boldsymbol{J}_{2}^{\mathrm{x}}
\end{array}\right)=-c_{\mathrm{t}} D^{\mathrm{x}} \cdot\left(\begin{array}{c}
\boldsymbol{\nabla} x_{1} \\
\boldsymbol{\nabla} x_{2}
\end{array}\right) .
$$

with the corresponding Fick diffusion matrix in the center of molar-velocity frame of reference

$$
\mathrm{D}^{\mathrm{x}}=\left[\begin{array}{ll}
D_{11}^{\mathrm{x}} & D_{12}^{\mathrm{x}} \\
D_{21}^{\mathrm{x}} & D_{22}^{\mathrm{x}}
\end{array}\right] .
$$

In contrast to a binary mixture where there is a single (scalar) diffusion coefficient, in ternary mixtures $\mathrm{D}^{\mathrm{w}} \neq \mathrm{D}^{\mathrm{x}}$. As mentioned in the introduction, this frame dependence of diffusion matrices has caused some difficulties in practice, when dealing with experimental data or numerical simulations. ${ }^{4,10}$ In this paper we propose a redefinition of Fick diffusion matrices that will eliminate this difficulty. In doing so, we build on a concept previously applied to thermodiffusion in ternary mixtures, where it was shown that the relationship between properties in the mass and in the molar frame of reference is easily expressed by introducing "concentration" matrices defined by ${ }^{19}$

$$
\mathbf{X}=\left[\begin{array}{cc}
x_{1}\left(1-x_{1}\right) & -x_{1} x_{2} \\
-x_{1} x_{2} & x_{2}\left(1-x_{2}\right)
\end{array}\right],
$$

and

$$
\mathbf{W}=\left[\begin{array}{cc}
w_{1}\left(1-x_{\mathrm{w}}\right) & -w_{1} w_{2} \\
-w_{1} w_{2} & w_{2}\left(1-w_{2}\right)
\end{array}\right] .
$$

In terms of these concentration matrices, the two gradients in mass fraction and the two gradients in mole fraction can be related by an expression that resembles eqn (7) for binary mixtures:

$$
\mathrm{X}^{-1} \cdot\left(\begin{array}{l}
\boldsymbol{\nabla} x_{1} \\
\boldsymbol{\nabla} x_{2}
\end{array}\right)=\mathrm{W}^{-1} \cdot\left(\begin{array}{c}
\boldsymbol{\nabla} w_{1} \\
\boldsymbol{\nabla} w_{2}
\end{array}\right) .
$$

From eqn (1) and (2) it can be shown that the two fluxes in the mole frame of reference are related to the two fluxes in the mass frame of reference by an expression that resembles eqn (5) for binary mixtures:

$$
\left(\begin{array}{l}
\boldsymbol{J}_{1}^{\mathrm{w}} \\
\boldsymbol{J}_{2}^{\mathrm{w}}
\end{array}\right)=\frac{\rho_{\mathrm{t}}}{c_{\mathrm{t}}} \mathrm{W} \cdot \mathbf{X}^{-1} \cdot\left(\begin{array}{l}
\boldsymbol{J}_{1}^{\mathrm{x}} \\
\boldsymbol{J}_{2}^{\mathrm{x}}
\end{array}\right) .
$$

Next, by combining eqn (17) and (18) with eqn (11) and (13), one readily obtains ${ }^{19}$

$$
\mathrm{W}^{-1} \cdot \mathrm{D}^{\mathrm{w}} \cdot \mathrm{W}=\mathrm{X}^{-1} \cdot \mathrm{D}^{\mathrm{x}} \cdot \mathrm{X} \equiv \mathrm{D},
$$


which defines a frame-independent diffusion matrix D. Of cause, use of this frame-independent diffusion matrix D implies a change of the actual formulation of Fick's law. In terms of the frame-independent diffusion matrix D, Fick's law in the mass frame of reference now reads:

$$
\left(\begin{array}{c}
\boldsymbol{J}_{1}^{\mathrm{w}} \\
\boldsymbol{J}_{2}^{\mathrm{w}}
\end{array}\right)=-\rho_{\mathrm{t}} \mathrm{W} \cdot \mathrm{D} \cdot \mathrm{W}^{-1} \cdot\left(\begin{array}{c}
\boldsymbol{\nabla} w_{1} \\
\boldsymbol{\nabla} w_{2}
\end{array}\right),
$$

while in the center of molar-velocity frame of reference Fick's law now reads:

$$
\left(\begin{array}{c}
\boldsymbol{J}_{1}^{\mathrm{x}} \\
\boldsymbol{J}_{2}^{\mathrm{x}}
\end{array}\right)=-c_{\mathrm{t}} \mathrm{X} \cdot \mathrm{D} \cdot \mathrm{X}^{-1} \cdot\left(\begin{array}{c}
\boldsymbol{\nabla} x_{1} \\
\boldsymbol{\nabla} x_{2}
\end{array}\right) .
$$

The advantage of this formulation of Fick's law is that exactly the same invariant diffusion matrix D appears in eqn (20) and in eqn (21). Notice from eqn (20) and (21) above that in the case of a binary mixture the product is commutative, so that the prefactors before and after the diffusion coefficient $D$ cancel and we recover Fick's law in its traditional form as given by eqn (6).

The ideas above can also be extended to include the volume frame of reference. In the case of the volume frame of reference we introduce a third concentration matrix defined by

$$
\Phi=\left[\begin{array}{cc}
x_{1}\left(1-\phi_{1}\right) & -x_{1} \phi_{2} \\
-\phi_{1} x_{2} & x_{2}\left(1-\phi_{2}\right)
\end{array}\right] .
$$

From eqn (2) and (3) it readily follows that for a ternary mixture

$$
\Phi^{-1} \cdot\left(\begin{array}{c}
\boldsymbol{J}_{1}^{\mathrm{V}} \\
\boldsymbol{J}_{2}^{\mathrm{V}}
\end{array}\right)=\mathrm{X}^{-1} \cdot\left(\begin{array}{c}
\boldsymbol{J}_{1}^{\mathrm{x}} \\
\boldsymbol{J}_{2}^{\mathrm{x}}
\end{array}\right),
$$

which represents the generalization of eqn (8) to a ternary mixture. Similarly to the role of matrices $\mathrm{W}$ and $\mathrm{X}$, the matrix $\Phi$ is convenient for relating gradients of mole fraction to the gradients of molar concentration. Indeed differentiation of the expression of molar concentration as a function of mole fraction yields:

$$
\left(\begin{array}{c}
\boldsymbol{\nabla} c_{1} \\
\nabla c_{2}
\end{array}\right)=c_{\mathrm{t}} \Phi \cdot \mathrm{X}^{-1} \cdot\left(\begin{array}{c}
\boldsymbol{\nabla} x_{1} \\
\boldsymbol{\nabla} x_{2}
\end{array}\right) .
$$

To obtain eqn (24) one needs to consider $c_{1}=x_{1} c_{\mathrm{t}}$ and $c_{2}=x_{2} c_{\mathrm{t}}$ and the expression of the molar volume of a ternary mixture:

$$
\hat{V}=x_{1} \hat{V}_{1}+x_{2} \hat{V}_{2}+x_{3} \hat{V}_{3}=c_{\mathrm{t}}^{-1} .
$$

In addition, one has to use the following thermodynamic relations linking the derivatives of the three partial molar volumes $\hat{V}_{i}$ of a ternary mixture:

$$
\begin{aligned}
& x_{1} \frac{\partial \hat{V}_{1}}{\partial x_{1}}+x_{2} \frac{\partial \hat{V}_{2}}{\partial x_{1}}+\left(1-x_{1}-x_{2}\right) \frac{\partial \hat{V}_{3}}{\partial x_{1}}=0, \\
& x_{1} \frac{\partial \hat{V}_{1}}{\partial x_{2}}+x_{2} \frac{\partial \hat{V}_{2}}{\partial x_{2}}+\left(1-x_{1}-x_{2}\right) \frac{\partial \hat{V}_{3}}{\partial x_{2}}=0,
\end{aligned}
$$

which apply when $x_{1}$ and $x_{2}$ are adopted as the two independent concentration variables and $x_{3}=1-x_{1}-x_{2}$.

Next, upon combining eqn (23) and (24) with Fick's law in the center of molar-velocity frame of reference, as given by eqn (21), we obtain in terms of the frame-invariant diffusion matrix D:

$$
\left(\begin{array}{c}
\boldsymbol{J}_{1}^{\mathrm{V}} \\
\boldsymbol{J}_{2}^{\mathrm{V}}
\end{array}\right)=-\Phi \cdot \mathrm{D} \cdot \Phi^{-1} \cdot\left(\begin{array}{c}
\boldsymbol{\nabla} c_{1} \\
\boldsymbol{\nabla} c_{2}
\end{array}\right),
$$

which is the natural generalization of eqn (9) for a binary mixture to a ternary mixture, and is consistent with eqn (20) or eqn (21). Note that the invariant Fick diffusion matrix D in eqn (27) for the molar diffusion fluxes relative to the center of volume velocity is identical to the one in eqn (20) for the mass diffusion fluxes relative to the center of mass velocity, and in eqn (21) for the molar diffusion fluxes relative to the center of molar velocity. Hence, we have indeed been able to formulate Fick's law for a ternary mixture in the three most useful frames of references in terms of a single invariant diffusion matrix D by applying a simple elegant similarity transformation. As a corollary to eqn (27), its combination with eqn (19) yields

$$
\mathrm{W}^{-1} \cdot \mathrm{D}^{\mathrm{W}} \cdot \mathrm{W}=\mathrm{X}^{-1} \cdot \mathrm{D}^{\mathrm{x}} \cdot \mathrm{X}=\Phi^{-1} \cdot \mathrm{D}^{\mathrm{V}} \cdot \Phi=\mathrm{D},
$$

where $\mathrm{D}^{\mathrm{V}}$ is the Fick diffusion matrix in the volume-average frame of ref. 6. Eqn (28) provides an alternative way of presenting the relation between Fick diffusion matrices in different reference frames, which in some circumstances may be advantageous compared with the more traditional way, as found, e.g., in the book of Taylor and Krishna. ${ }^{6}$

There are some differences between the matrix $\Phi$ on the one hand and the matrices $\mathrm{W}$ and $\mathrm{X}$ on the other hand that are relevant from a practical point of view. Since laboratory mixtures are typically prepared by weight, the matrices $\mathrm{W}$ and $\mathrm{X}$ are commonly known. However, a full knowledge of the matrix $\Phi$ requires equations of state for the partial molar volumes $\hat{V}_{i}$. We also note that the matrices $\mathrm{W}$ and $\mathrm{X}$ are symmetric, while the matrix $\Phi$ is not.

We conclude this section with some comments on the advantages and disadvantages of frame-invariant Fick diffusion matrices versus frame-invariant Maxwell-Stefan diffusion matrices. Maxwell-Stefan diffusion matrices relate diffusion fluxes to chemical potential gradients. ${ }^{11-14}$ They are convenient for molecular dynamics simulations, since they are related to time-dependent correlation functions. ${ }^{14,24,25}$ Another advantage of Maxwell-Stefan matrices is that they are symmetric, while the frame-invariant Fick diffusion matrices D are not. However, the frame-invariant Fick diffusion matrices are directly related to the concentrations and concentration gradients that are more easily accessible experimentally. Hence, we believe that the frame-invariant Fick diffusion matrices, introduced in this paper, will be more convenient in practice for the interpretation and intercomparison of experimental diffusion data. The relations between Maxwell-Stefan diffusion matrices and frame-dependent Fick diffusion matrices $\mathrm{D}^{\mathrm{w}}$ and $\mathrm{D}^{\mathrm{x}}$ have been discussed in the literature. ${ }^{14,24,27}$ of course, to relate 
Maxwell-Stefan diffusion matrices to the frame-invariant Fick diffusion matrices an additional similarity transformation in accordance with eqn (19) is required.

\section{Binary limits of ternary mixtures}

For dealing with diffusion in ternary mixtures it is useful to discuss the diffusion matrices in the three binary-component limits, that is, at the sides of the ternary concentration triangle, where one of the concentrations is dilute. For diffusion matrices in the barycentric frame of reference the limiting relations are given by: $:^{5,28}$

$$
\begin{aligned}
& \text { when } x_{1} \rightarrow 0,\left\{\begin{array}{l}
D_{22}^{\mathrm{w}} \rightarrow D_{\text {bin }}^{(23)} \\
D_{12}^{\mathrm{w}} \rightarrow 0
\end{array}\right. \\
& \text { when } x_{2} \rightarrow 0,\left\{\begin{array}{l}
D_{11}^{\mathrm{w}} \rightarrow D_{\text {bin }}^{(13)} \\
D_{21}^{\mathrm{w}} \rightarrow 0
\end{array}\right. \\
& \text { when } x_{3} \rightarrow 0,\left\{\begin{array}{l}
D_{22}^{\mathrm{w}} \rightarrow D_{21}^{\mathrm{w}} \rightarrow D_{\text {bin }}^{(12)} \\
D_{11}^{\mathrm{w}}+D_{21}^{\mathrm{w}}-D_{12}^{\mathrm{w}}-D_{22}^{\mathrm{w}} \rightarrow 0
\end{array}\right.
\end{aligned}
$$

where $D_{\text {bin }}^{(i j)}$ represents the diffusion coefficient in the binary mixture with components $i$ and $j$. In a binary mixture the single diffusion coefficient $D_{\text {bin }}^{(i j)}$ is frame independent, so no further notation is needed. Since some of the diffusion coefficients in ternary mixtures are typically measured with much higher accuracy than others, eqn (29) turn out to be useful in practice. ${ }^{5}$ It is, therefore, interesting to find the corresponding limits for the frame-invariant diffusion matrix D defined by eqn (19). Combining eqn (29) and (19) with the explicit expressions, eqn (15) and (16), of the concentration matrices, one finds after some algebra:

$$
\begin{aligned}
& \text { when } x_{1} \rightarrow 0,\left\{\begin{array}{l}
D_{22} \rightarrow D_{\text {bin }}^{(23)} \\
D_{12} \rightarrow 0
\end{array},\right. \\
& \text { when } x_{2} \rightarrow 0,\left\{\begin{array}{l}
D_{11} \rightarrow D_{\text {bin }}^{(13)} \\
D_{12} \rightarrow 0
\end{array},\right. \\
& \text { when } x_{3} \rightarrow 0,\left\{\begin{array}{l}
D_{11}-D_{21} \rightarrow D_{\text {bin }}^{(12)} \\
D_{11}-D_{21}+D_{12}-D_{22} \rightarrow 0
\end{array}\right.
\end{aligned}
$$

It is interesting to compare the binary limits (29) of the framedependent diffusion matrices with the binary limits (30) of the frame-independent diffusion matrix. The limits for the main diffusivities are similar, whereas cross-diffusivities show different behaviors. For instance, in the limit $x_{1} \rightarrow 0$ the framedependent cross-diffusivity $D_{12}^{\mathrm{w}}$ must vanish, while in the same limit it is the frame-independent cross-diffusivity $D_{21}$ that vanishes.

\section{Composition dependence of Fick diffusion matrices}

As is the case for thermodiffusion coefficients in binary mixtures, ${ }^{18,29}$ one may expect that in mixtures of weakly interacting components the concentration prefactors in our formulation of Fick's law may carry a significant part of the composition dependence, so that the frame-invariant diffusion matrix D in eqn (28) could become less dependent on concentration than the corresponding Fick diffusion matrices in the various reference frames.

Obviously, a detailed clarification of this issue requires an extensive and thorough investigation. However, for the sake of a simple illustration, we have analyzed the extensive data set obtained by Sechenyh et al., who measured the diffusion matrix in the barycentric frame of reference for the mixture 1,2,3,4-tetrahydronaphthalene + isobutylbenzene $+n$-dodecane at 20 different compositions. ${ }^{5}$ For each of the experimental compositions we have evaluated the corresponding frame-invariant diffusion matrix D defined by eqn (19). The results of this analysis for the cross-diffusivities are displayed as ternary contour plots in Fig. 1. The left column shows the plots for the barycentric crossdiffusivities $D_{12}^{\mathrm{w}}$ and $D_{21}^{\mathrm{w}}$ reported by Sechenyh et al. The right column shows the corresponding frame-independent crossdiffusivities obtained from eqn (19). Note that the color scheme is the same for the plots in the left and right columns. One sees that the intermediate greenish colors dominate In larger areas in the plots in the right column, while the contour curves are generally more separated, indicating that composition dependence is less important for the frame-invariant diffusivities. In particular, the two local minima appearing in the plots of $D_{12}^{\mathrm{w}}$ and $D_{21}^{\mathrm{w}}$ do not show up in their corresponding frameindependent counter parts. Note also that in the frame invariant cases the limits of eqn (30) are very smoothly approached, either at $w_{1} \rightarrow 0$ for $D_{21}$ or at $w_{2} \rightarrow 0$ for $D_{12}$.

\section{Isothermal Fick diffusion in multicomponent mixtures}

For simplicity we started by formulating frame-invariant Fick diffusion matrices in detail for ternary mixtures in Section 4 . However, the procedure can be readily extended to mixtures with an arbitrary number of components. For this purpose we consider $(n-1) \times(n-1)$ matrices $\mathrm{X}, \mathrm{W}$, and $\Phi$ with matrix elements defined by

$X_{i j}=x_{i} \delta_{i j}-x_{i} x_{j}, \quad W_{i j}=w_{i} \delta_{i j}-w_{i} w_{j}, \quad \Phi_{i j}=x_{i} \delta_{i j}-\phi_{j} x_{i}$.

Here $i, j \in\{1, n-1\}$ are the independent components, while $x_{n}$, $w_{n}$, or $\phi_{n}$ are the dependent concentrations, and where $\delta_{i j}$ are Kronecker deltas. Then a little bit of algebra shows that eqn (17) 

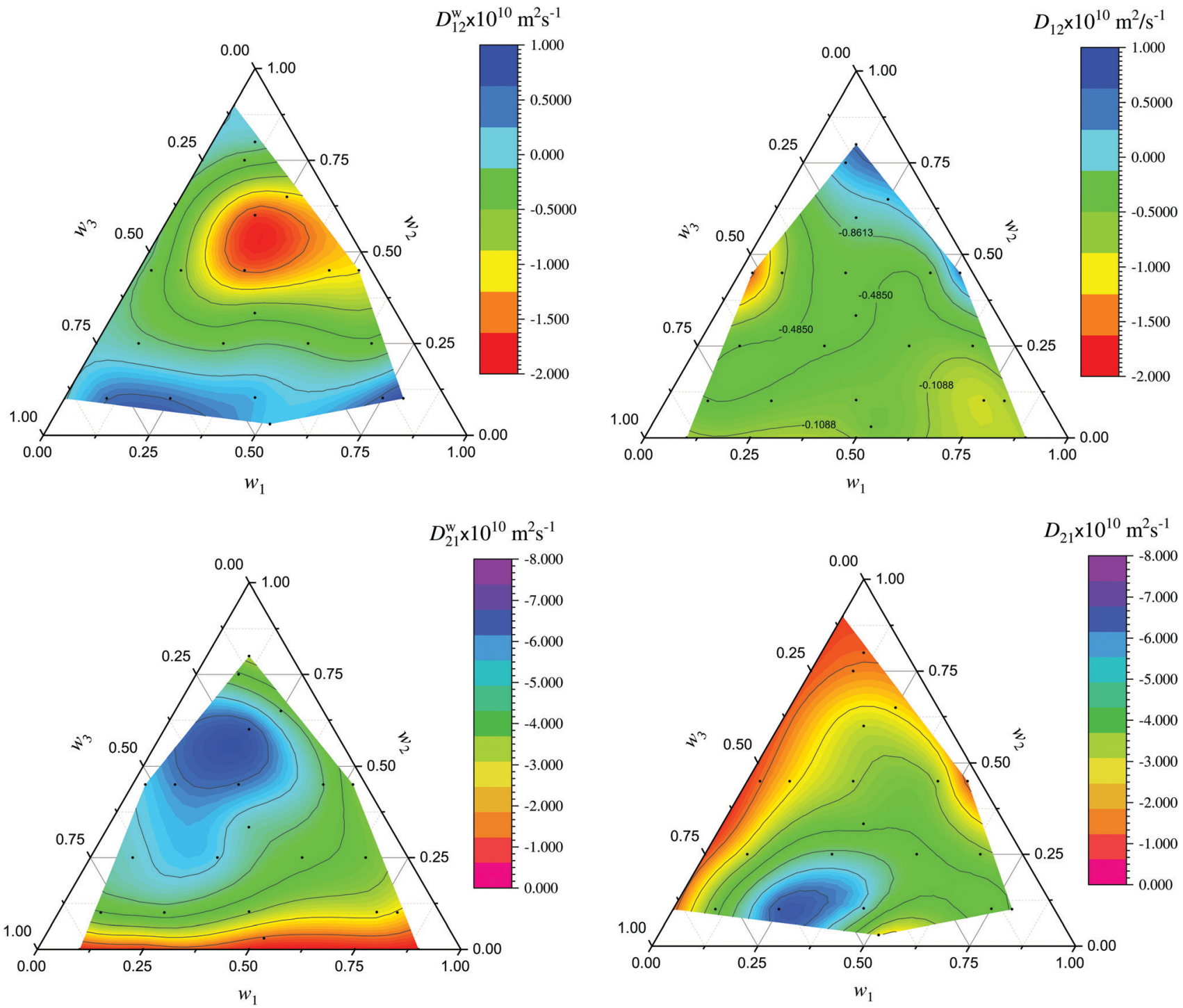

Fig. 1 Ternary contour plots of the frame-dependent cross-diffusivities $D_{12}^{w}$ and $D_{21}^{w}$ (left column) for a mixture of 1,2,3,4-tetrahydronaphthalene + isobutylbenzene $+n$-dodecane reported by Sechenyh et al., ${ }^{5}$ and the corresponding frame-independent cross-diffusivities $D_{12}$ and $D_{21}$ obtained from eqn (19). The binary limits, given by eqn (29) or eqn (30), have been used to complement the experimental data in each case.

holds in general:

$$
\mathrm{X}^{-1} \cdot\left(\begin{array}{c}
\boldsymbol{\nabla} x_{1} \\
\boldsymbol{\nabla} x_{2} \\
\vdots \\
\boldsymbol{\nabla} x_{n-1}
\end{array}\right)=\mathrm{W}^{-1} \cdot\left(\begin{array}{c}
\boldsymbol{\nabla} w_{1} \\
\boldsymbol{\nabla} w_{2} \\
\vdots \\
\boldsymbol{\nabla} w_{n-1}
\end{array}\right) .
$$

Similarly, eqn (24) for molar concentrations also holds in general:

$$
\Phi^{-1} \cdot\left(\begin{array}{c}
\boldsymbol{\nabla} c_{1} \\
\boldsymbol{\nabla} c_{2} \\
\vdots \\
\nabla c_{n-1}
\end{array}\right)=c_{\mathrm{t}} \mathrm{X}^{-1} \cdot\left(\begin{array}{c}
\boldsymbol{\nabla} x_{1} \\
\boldsymbol{\nabla} x_{2} \\
\vdots \\
\boldsymbol{\nabla} x_{n-1}
\end{array}\right)
$$

The relations (18) and (23) between the diffusion fluxes in different reference frames generalize to:

$$
\left(\begin{array}{c}
\boldsymbol{J}_{1}^{\mathrm{w}} \\
\boldsymbol{J}_{2}^{\mathrm{w}} \\
\vdots \\
\boldsymbol{J}_{n-1}^{\mathrm{w}}
\end{array}\right)=\frac{\rho_{\mathrm{t}}}{c_{\mathrm{t}}} \mathrm{W} \cdot \mathrm{X}^{-1} \cdot\left(\begin{array}{c}
\boldsymbol{J}_{1}^{\mathrm{x}} \\
\boldsymbol{J}_{2}^{\mathrm{X}} \\
\vdots \\
\boldsymbol{J}_{n-1}^{\mathrm{x}}
\end{array}\right),
$$

and

$$
\left(\begin{array}{c}
\boldsymbol{J}_{1}^{\mathrm{V}} \\
\boldsymbol{J}_{2}^{\mathrm{V}} \\
\vdots \\
\boldsymbol{J}_{n-1}^{\mathrm{V}}
\end{array}\right)=\Phi \cdot \mathrm{X}^{-1} \cdot\left(\begin{array}{c}
\boldsymbol{J}_{1}^{\mathrm{x}} \\
\boldsymbol{J}_{2}^{\mathrm{x}} \\
\vdots \\
\boldsymbol{J}_{n-1}^{\mathrm{x}}
\end{array}\right)
$$


Hence, the $(n-1) \times(n-1)$ matrices $\mathrm{X}, \mathrm{W}$, and $\Phi$ can be used to define a frame-independent Fick diffusion matrix $\mathrm{D}$ for multicomponent mixtures just in the same way as was shown explicitly for ternary mixtures in Section 4. Specifically, the Fick's laws, given by eqn (20), (21) or (27) for ternary mixtures in Section 4, hold in general for an arbitrary number of components with a single frame-invariant diffusion matrix D:

$$
\begin{gathered}
\left(\begin{array}{c}
\boldsymbol{J}_{1}^{\mathrm{w}} \\
\boldsymbol{J}_{2}^{\mathrm{w}} \\
\vdots \\
\boldsymbol{J}_{n-1}^{\mathrm{w}}
\end{array}\right)=-\rho_{t} \mathrm{~W} \cdot \mathrm{D} \cdot \mathrm{W}^{-1} \cdot\left(\begin{array}{c}
\boldsymbol{\nabla} w_{1} \\
\boldsymbol{\nabla}_{w_{2}} \\
\vdots \\
\boldsymbol{\nabla}_{w_{n-1}}
\end{array}\right), \\
\left(\begin{array}{c}
\boldsymbol{J}_{1}^{\mathrm{x}} \\
\boldsymbol{J}_{2}^{\mathrm{x}} \\
\vdots \\
\boldsymbol{J}_{n-1}^{\mathrm{x}}
\end{array}\right)=-c_{\mathrm{t}} \mathrm{X} \cdot \mathrm{D} \cdot \mathrm{X}^{-1} \cdot\left(\begin{array}{c}
\boldsymbol{\nabla} x_{1} \\
\boldsymbol{\nabla} x_{2} \\
\vdots \\
\boldsymbol{\nabla} x_{n-1} \\
\boldsymbol{\nabla}_{c_{1}} \\
\boldsymbol{\nabla}_{c_{2}}^{\mathrm{V}} \\
\vdots \\
\boldsymbol{J}_{2}^{\mathrm{V}} \\
\vdots \\
\boldsymbol{J}_{n-1}^{\mathrm{V}}
\end{array}\right),
\end{gathered}
$$

As a corollary, eqn (28) continues to hold for an arbitrary number of components providing an alternative way of switching Fick diffusion matrices between different reference frames.

\section{Non-isothermal diffusion in multicomponent mixtures}

When diffusion is not isothermal, there is a thermodiffusion contribution proportional to the temperature gradient $\nabla$. When both a concentration gradient and a temperature gradient are present, the molar diffusion flux in a binary mixture has traditionally been expressed as ${ }^{13,21}$

$$
J^{\mathrm{x}}=-c_{\mathrm{t}}\left[D \nabla x+x(1-x) D_{\mathrm{T}} \boldsymbol{\nabla} T\right],
$$

while the mass diffusion flux is expressed as

$$
J^{\mathrm{w}}=-\rho_{\mathrm{t}}\left[D \nabla w+w(1-w) D_{\mathrm{T}} \boldsymbol{\nabla} T\right] .
$$

Note the presence of the concentration prefactors, $x(1-x)$ in eqn (39) and $w(1-w)$ in eqn (40). They are used so that the thermodiffusion coefficient $D_{\mathrm{T}}$ has the same value in eqn (39) and in eqn (40). Essentially we have used the same concept in defining frame-independent Fick diffusion matrices in the contribution from the concentration gradient. In the previous paper, it has been shown how the thermodiffusion contribution proportional to the temperature gradient can be formulated for a multicomponent mixture in terms of $(n-1)$ frameindependent thermodiffusion coefficients $D_{\mathrm{T}, i}(i \in 1, n-1) .{ }^{19}$
Combining the formulation developed for the Fick diffusion contribution in this paper with that for the thermodiffusion contribution, we thus obtain for a mixture of $n$ components:

$$
\left(\begin{array}{c}
\boldsymbol{J}_{1}^{\mathrm{x}} \\
\boldsymbol{J}_{2}^{\mathrm{x}} \\
\vdots \\
\boldsymbol{J}_{n-1}^{\mathrm{x}}
\end{array}\right)=-c_{\mathrm{t}}\left(\mathrm{X} \cdot \mathrm{D} \cdot \mathrm{X}^{-1} \cdot\left(\begin{array}{c}
\boldsymbol{\nabla} x_{1} \\
\boldsymbol{\nabla} x_{2} \\
\vdots \\
\boldsymbol{\nabla}_{x_{n-1}}
\end{array}\right)+\mathrm{X} \cdot\left(\begin{array}{c}
D_{\mathrm{T}, 1} \\
D_{\mathrm{T}, 2} \\
\vdots \\
D_{\mathrm{T}, n-1}
\end{array}\right) \boldsymbol{\nabla T}\right\} \text {, }
$$

and

$$
\left(\begin{array}{c}
\boldsymbol{J}_{1}^{\mathrm{w}} \\
\boldsymbol{J}_{2}^{\mathrm{w}} \\
\vdots \\
\boldsymbol{J}_{n-1}^{\mathrm{w}}
\end{array}\right)=-\rho_{\mathrm{t}}\left\{\mathrm{W} \cdot \mathrm{D} \cdot \mathrm{W}^{-1} \cdot\left(\begin{array}{c}
\boldsymbol{\nabla} w_{1} \\
\boldsymbol{\nabla}_{w_{2}} \\
\vdots \\
\boldsymbol{\nabla}_{w_{n-1}}
\end{array}\right)+\mathrm{W} \cdot\left(\begin{array}{c}
D_{\mathrm{T}, 1} \\
D_{\mathrm{T}, 2} \\
\vdots \\
D_{\mathrm{T}, n-1}
\end{array}\right) \boldsymbol{\nabla} T\right\} .
$$

\section{Conclusions}

We have shown, first for a ternary mixture and subsequently for a multicomponent mixture, how the diffusion resulting from a concentration gradient, can be expressed in terms of a Fick diffusion matrix D, defined by eqn (28), that is independent of the frame of reference. When combined with thermodiffusion resulting from a temperature gradient, we have obtained a formulation that contains both a frame-independent diffusion matrix $\mathrm{D}$ and $n-1$ frame-independent thermodiffusion coefficients $D_{\mathrm{T}, i}$ as shown in eqn (41) and (42).

\section{Conflicts of interest}

There are no conflicts of interest.

\section{Acknowledgements}

We thank Aliaksandr Mialdun from the Universite Libre de Bruxelles for a careful review of the manuscript. We also thank Dick Bedeaux of the Norwegian University of Science and Technology in Trondheim for valuable discussions. We acknowledge support from the European Space Agency (ESA) through the TechNEs MAP project. The research at the Complutense University was further supported by grant ESP201783544-C3-2-P of the Spanish Agencia Estatal de Investigación.

\section{References}

1 J. C. Legros, Y. Gaponenko, A. Mialdun, T. Triller, A. Hammon, C. Bauer, W. Köhler and V. Shetsova, Phys. Chem. Chem. Phys., 2015, 41, 27713-27725.

2 M. Larrañaga, M. M. Bou-Ali, I. Lizarraga, J. A. Madariaga and C. Santamaría, J. Chem. Phys., 2015, 143, 024202. 
3 M. M. Bou-Ali, A. Ahadi, D. Alonso de Mezquia, Q. Galand, M. Gebhardt, O. Khlybov, W. Köhler, M. Larrañaga, J. C. Legros, T. Lyubimova, A. Mialdun, I. Ryzhkov, M. Z. Saghir, V. Shetsova and S. Van Vaerenbergh, Eur. Phys. J. E: Soft Matter Biol. Phys., 2015, 38, 30.

4 A. Mialdun, V. Sechenyh, J. C. Legros, J. M. Ortiz de Zárate and V. Shetsova, J. Chem. Phys., 2013, 139, 104903.

5 V. Sechenyh, J. C. Legros, A. Mialdun, J. M. Ortiz de Zárate and V. Shetsova, J. Phys. Chem. B, 2016, 120, 535-548.

6 R. Taylor and R. Krishna, Multicomponent Mass Transfer, Wiley, New York, 1993.

7 T. Groszman and J. Winkelmann, J. Chem. Eng. Data, 2009, 54, 405-410.

8 T. Groszman and J. Winkelmann, J. Chem. Eng. Data, 2009, 54, 485-490.

9 G. B. Bay and D. G. Leaist, J. Chem. Eng. Data, 2010, 55, 1814-1820.

10 S. Kozlova, A. Mialdun, I. Ryzhkov, T. Janzen, J. Vrabec and V. Shevtsova, Phys. Chem. Chem. Phys., 2019, 21, 2140-2152.

11 G. D. C. Kuiken, Thermodynamics of Irreversible Processes, Wiley, New York, 1994.

12 R. Krishna and J. A. Wesselingh, Chem. Eng. Sci., 1997, 52, 861-911.

13 V. Demirel, Nonequilibrium Thermodynamics, Elsevier, Amsterdam, 3rd edn, 2014.

14 S. Kjelstrup, D. Bedeaux, E. Johannessen and J. Gross, NonEquilibrium Thermodynamics for Engineers, World Scientific, Singapore, 2010, ch. 5.

15 C. Peters, L. Wolff, T. J. H. Vlught and A. Bardow, in Experimental Thermodynamics Vol. X, Non-Equilibrium Thermodynamics with Applications, ed. D. Bedeaux, S. Kjelstrup and J. V. Sengers, Royal Society of Chemistry, Cambridge, 2016, ch. 5.
16 R. B. Bird, W. E. Stewart and E. N. Lightfood, Transport Phenomena, Wiley, New York, 2nd edn, 2002.

17 D. A. Drew and S. L. Passman, Theory of Multicomponent Fluids, Springer, Berlin, 1999.

18 W. Köhler and K. I. Morozov, J. Non-Equilib. Thermodyn., 2016, 41, 151-197.

19 J. M. Ortiz de Zárate, Eur. Phys. J. E: Soft Matter Biol. Phys., 2019, 42, 43.

20 S. R. de Groot and P. Mazur, Non-Equilibrium Thermodynamics, North-Holland, Amsterdam, 1962.

21 G. Lebon, D. Jou and J. Casas-Vázquez, Understanding Nonequilibrium Thermodynamics, Springer, Berlin, 2008.

22 G. Galliero, H. Bataller, J.-P. Bazile, J. Diaz, F. Croccolo, H. Hoang, R. Vermorel, P.-A. Artola, B. Rousseau, V. Vesovic, M. M. Bou-Ali, J. M. Ortiz de Zárate, S. Xu, K. Zhang, F. Montel, A. Verga and O. Minster, npj Microgravity, 2017, 3, 20.

23 M. C. Barros, A. C. F. Ribeiro, L. M. P. Verissimo, D. G. Leaist and M. A. Esteso, J. Chem. Thermodyn., 2018, 123, 17-21.

24 X. Liu, A. Martín-Calvos, E. McGarrity, S. K. Schnell, S. Calero, J.-M. Simon, D. Bedeaux, S. Kjelstrup, A. Bardow and T. J. H. Vlugt, Ind. Eng. Chem. Res., 2012, 51, 10247-10258.

25 X. Liu, S. K. Schnell, J.-M. Simon, P. Krüger, D. Bedeaux, S. Kjelstrup, A. Bardow and T. J. H. Vlugt, Int. J. Thermophys., 2013, 34, 1169-1196.

26 J. A. Bard, J. G. Albright, D. G. Miller and M. E. Zeidler, J. Chem. Soc., Faraday Trans., 1996, 92, 4187-4197.

27 Y. Demirel and V. Gerbaud, Nonequilibrium Thermodynamics: Transport and Rate Processes in Physical, Chemical, and Biological Processes, Elsevier, Amsterdam, 4th edn, 2019, ch. 6.

28 P. K. Gupta and A. R. Cooper, Jr., Physica, 1971, 54, 39-59.

29 S. Hartmann, G. Wittko, F. Schock, W. Grosz, F. Lindner, W. Köhler and K. I. Morozov, J. Chem. Phys., 2014, 141, 14503. 